\title{
First-cycle absolute neutrophil count can be used to improve chemotherapy-dose delivery and reduce the risk of febrile neutropenia in patients receiving adjuvant therapy: a validation study
}

\author{
Edgardo Rivera ${ }^{1}$, M Haim Erder ${ }^{2}$, Moshe Fridman $^{3}$, Debra Frye ${ }^{1}$ and Gabriel N Hortobagyi ${ }^{1}$ \\ ${ }^{1}$ University of Texas, MD Anderson Cancer Center, Houston, Texas, USA \\ ${ }^{2}$ Amgen Inc, Thousand Oaks, California, USA \\ ${ }^{3}$ AMF Consulting Inc., Los Angeles, California, USA
}

Corresponding author: Edgardo Rivera (e-mail: erivera@mail.mdanderson.org)

Received: 11 Mar 2003 Accepted: 4 Jun 2003 Published: 20 Jun 2003

Breast Cancer Res 2003, 5:R114-R120 (DOI 10.1186/bcr618)

(c) 2003 Rivera et al., licensee BioMed Central Ltd (Print ISSN 1465-5411; Online ISSN 1465-542X). This is an Open Access article: verbatim copying and redistribution of this article are permitted in all media for any purpose, provided this notice is preserved along with the article's original URL.

\begin{abstract}
Background: The nadir value of the absolute neutrophil count (ANC) in the first cycle of chemotherapy is an effective predictor of subsequent neutropenic events. This study was designed to validate an earlier published study based on a retrospective data analysis from a prospective randomized clinical trial.

Methods: The original published model was applied to a trial of 143 patients to cross-validate the model. We also tested the specification of the model on our data by using a logistic regression model with several variables, including first-cycle nadir ANC, age, menopausal status, hormone-receptor status, previous radiotherapy, and first-cycle decrease in hemoglobin concentration. Patients received fluorouracil, doxorubicin, and cyclophosphamide every 21 or 28 days for six cycles without hematopoietic support from colonystimulating factor.
\end{abstract}

Results: In the cross-validation analysis, the original model successfully classified patients by risk of neutropenic events $(C=0.78)$. When the model specification was tested, first-cycle nadir ANC was the sole significant $(P<0.0001)$ predictor of neutropenic events and the model had a good predictive power $(C=0.78)$. The estimated relative risk of 4.8 did not differ from the risk cited in the original model $(P=0.91)$. A significantly higher percentage of our patients with a low first-cycle nadir ANC of $0.25 \times 10^{9} /$ liter or less experienced febrile neutropenia (30\% versus $10 \%, P=0.04$ ) and received at least $85 \%$ of the planned dose intensity (55\% versus $32 \%, P=0.05)$.

Conclusions: The original risk model used to predict neutropenic events was validated by our study. This information can be used to target high-risk patients for prophylactic treatment with filgrastim (recombinant methionyl human granulocyte colonystimulating factor) in chemotherapy cycles 2 to 6 .

Keywords: chemotherapy, filgrastim, hospitalization, myelosuppression, neutropenia

\section{Introduction}

Neutropenic complications, defined as febrile neutropenia, severe neutropenia, or dose delay or reduction due to neutropenia, are the most common side effects of myelosuppressive chemotherapy $[1,2]$. The frequent occurrence of neutropenic complications suggests that it is impossible to predict accurately which patients will present with a neutropenic complication. Febrile neutropenia, defined as an absolute neutrophil count (ANC) of more than $1.0 \times 10^{9} /$ liter with a temperature of more than $100.6^{\circ} \mathrm{F}$, is the most severe neutropenic complication and can cause prolonged hospitalization. Neutropenic complications can negatively affect the course of chemotherapy, leading to dose delays or decreases to reduce a patient's risk of developing febrile neutropenia. The ability to improve predictions of which patients are at risk for neutropenic complications

ANC = absolute neutrophil count; CAF = chemotherapy with cyclophosphamide, doxorubicin, fluorouracil; MDACC = MD Anderson Cancer Center. 
might help to reduce the morbidity due to febrile neutropenia and increase the likelihood of delivering full chemotherapy dose on time. In addition, hematopoietic growth factors could be administered to patients who need them most, permitting the more efficient use of medical resources.

Several publications have argued that the first chemotherapy cycle nadir ANC is a good predictor of neutropenic complications in patients with breast cancer $[1,2]$. These papers were based on retrospective chart analyses and therefore require further validation in additional chemotherapy regimens and patient populations before the model can be prospectively implemented.

Filgrastim (recombinant methionyl human granulocyte colony-stimulating factor) has been shown to reduce the depth of the nadir ANC, shorten the duration of neutropenia, and reduce the risk of febrile neutropenia [3], thereby maintaining chemotherapy dose [4,5]. Many patients with early-stage breast cancer do not receive the full chemotherapy dose intensity recommended in conventional adjuvant chemotherapy regimens [6-8]. Neutropenia is the primary reason for chemotherapy dose delays and reductions $[1,6,7]$.

Dose delay and dose reduction can result in care that is less than optimal. Significant reductions in total dose and dose intensity have an adverse effect on disease-free survival and overall survival $[9,10]$. Because most dose delays and reductions occur after a neutropenic complication or febrile neutropenia, patients might experience an infectious episode and might need to contend with the burden and anxiety associated with a hospitalization and the risk of prolonged complications in treatment. These events impose potentially preventable burdens on the patients. The use of hematopoietic growth factors as primary prophylaxis is one way of decreasing the burden of neutropenic complications. However, when growth factors are not used as primary prophylaxis, neutropenic complications serve as the indicator of the patient's risk for further neutropenic complications, resulting either in growth-factor treatment as secondary prophylaxis or in chemotherapy dose delays or reductions.

An alternative treatment strategy can be developed in which patients have a reduced risk of neutropenic complications and a higher likelihood of receiving a full dose of chemotherapy on time. First-cycle nadir ANC can be used to identify patients at risk for neutropenic complications. These patients could receive prophylactic hematopoietic growth-factor support to decrease treatment-related morbidity and to increase the likelihood of the administration of full-dose chemotherapy on time.

Silber and colleagues [1] proposed a predictive model of neutropenia, dose reduction, or dose delay based on blood counts observed during the first cycle of chemotherapy. The model estimates the patient's risk of requiring dose attenuation due to myelosuppression or, alternatively, decreasing below acceptable ANC levels in subsequent chemotherapy cycles. The model demonstrates that patients can be ranked by probability of presenting with neutropenia in subsequent cycles with a high degree of certainty and good degree of discrimination between patients at high and low risk. This ranking permits the judicious use of hematopoietic growth factors, such as filgrastim, to support patients who can benefit the most from this treatment. Consequently, patients at high risk for developing neutropenia will have a greater chance of receiving full-dose chemotherapy on time and avoiding febrile neutropenia.

The purpose of our study was the further validation of a model $[1,11]$ that used first-cycle nadir ANC count to predict the risk of febrile neutropenia, dose delay, and dose reduction in patients with breast cancer receiving myelosuppressive chemotherapy. The validation had two parts: a cross-validation of the original model with our data and a test of the model specification to improve its predictive power for our specific patient population and regimen.

\section{Methods \\ Patients}

The validation sample included 153 chemotherapy-naive patients with stage I, II, IIla, or IIIb breast cancer who had been treated with adjuvant chemotherapy during 1986-92 as part of the MD Anderson Cancer Center (MDACC) 86-12 trial. Patients aged more than 75 years, those with inflammatory breast cancer, those with a previous history of contralateral breast cancer with higher stage of disease, and those with second primary other than basal cell carcinoma of skin or in situ carcinoma of cervix were excluded from the original MDACC 86-12 study.

Of the 153 patients participating in the trial, 10 were excluded from analysis. Four patients had no complete blood cell count data recorded, three had incomplete chemotherapy delivery information, and three had received filgrastim. Results are reported for the remaining 143 patients. Therefore, as in the study by Silber and colleagues [1], this validation sample describes the natural history of neutropenia.

\section{Treatment}

Primary surgical treatment consisted of a modified radical mastectomy or segmental mastectomy. If indicated, the patient was treated with preoperative or postoperative local radiotherapy. All patients were treated with the FAC (fluorouracil, doxorubicin, cyclophosphamide) regimen that consisted of $50 \mathrm{mg} / \mathrm{m}^{2}$ doxorubicin given as a continuous infusion over 72 hours starting on day $1,500 \mathrm{mg} / \mathrm{m}^{2}$ 
cyclophosphamide intravenously on day 1 , and $500 \mathrm{mg} / \mathrm{m}^{2}$ fluorouracil intravenously on days 1 and 4 . Treatment was given every 3 or 4 weeks for a total of six cycles. Complete blood counts with differential counts were usually obtained on days 1,8 , and 15 of each cycle. Chemotherapy was usually delayed if the ANC was less than $1.5 \times 10^{9} /$ liter on the planned first day of the cycle. Dose delays were usually for 1 week. The chemotherapy dose was reduced if, on the previous cycle, the nadir ANC was less than $0.5 \times 10^{9} /$ liter or if the patient experienced an episode of febrile neutropenia, defined as an ANC of less than $1.0 \times 10^{9} /$ liter with a temperature of more than $100.6^{\circ} \mathrm{F}$. Dose reductions usually consisted of a $20 \%$ reduction in dosage of all chemotherapy drugs.

\section{Outcome definition}

A neutropenia-related event was defined similarly to that in the study by Silber and colleagues [1]: a nadir ANC of $0.5 \times 10^{9} /$ liter or less, a neutropenia-related dose reduction of $15 \%$ or more of the planned dose of any agent, a neutropenia-related dose delay of 7 days or more, or a febrile-neutropenic episode. Delays and reductions were related to neutropenia, and the reasons for the dose modifications were documented. A response group for the risk model consists of all patients who experienced at least one neutropenia-related event after the first cycle of chemotherapy. The ANC cutoff point to define an event was $0.5 \times 10^{9} /$ liter, rather than $0.25 \times 10^{9} /$ liter used in the Silber model to accommodate the physician practice style associated with patients in the validation sample.

\section{Statistical methods}

The originally estimated Silber risk model was cross-validated with the MDACC sample. We used the same definitions as in the original model for the predictor variables. The distribution of these predictor variables was compared across the original Silber and validation MDACC study samples. The outcome variable in the validation sample differs in that an ANC cutoff point of $0.5 \times 10^{9} /$ liter is used in the compound outcome definition. Given this difference in ANC cutoff point and the difference in chemotherapy regimens used in the two samples, the absolute predicted probabilities of events resulting from the cross-validation might not be interpretable, but their relative magnitude (or ranking of patients by predicted probability of an event) is valid. To assess the cross-validation results, we divided the patients into predicted positive and predicted negative groups by using varying probability cut points. This process is equivalent to ranking the patients by their predicted event probability and dividing the ranked list in all possible ways, declaring the higherranked patients predicted positive and the lower-ranked patients predicted negative.

The sensitivity and specificity of each possible resulting acteristic curve plot. The $C$-statistic (area under the receiver-operating-characteristic curve) [12], which summarized the sensitivity and specificity across the entire (0 to 1) range of probability cut points, is reported together with the sensitivity and specificity rates for the optimal classification table. This validation process does not address the effect of concurrent radiotherapy because none of the patients in the validation sample were treated with this modality. As a result, the indicator variable for concurrent radiotherapy present in the original model is set to 0 , effectively applying the model to patients with no concurrent radiotherapy.

For the model-specification test, the same variables used to predict neutropenic complications in the Silber model were fitted with a logistic regression to our data and the significance was reported. Furthermore, we explored other potential predictors to examine whether new significant risk factors would emerge. The discrimination and calibration of the model fitted to our data and original models were compared by using the $\mathrm{C}$-statistic and the Hosmer-Lemeshow statistic [13]. The receiver-operatingcharacteristic curves for the two models were plotted and compared. The magnitudes of the risk factors' effects were compared across the validation and original models. Regression coefficient differences were tested with an asymptotic normal test and the estimated probability of event curves derived from the original and validation models were plotted for comparison. Finally, we assessed the importance of first-cycle nadir ANC as a predictor of subsequent episodes of febrile neutropenia and of reduced dose intensity (not more than $85 \%$ of planned dose intensity) by comparing the proportion of observed events in patients grouped by first-cycle nadir ANC ranges. Statistical analyses were performed by using SAS 8.00 for Windows (SAS Institute Inc, Cary, NC). All reported $P$ values are two-sided.

\section{Results \\ Patients}

Patient characteristics in the validation sample were similar to those described in the Silber model [1], except for the distribution of patients with positive nodes (Table 1). In Silber's patient sample, one-third of the patients were treated with the less myelosuppressive CMF (cyclophosphamide, methotrexate, fluorouracil) chemotherapy regimen, and the CAF (cyclophosphamide, doxorubicin, fluorouracil) regimen consisted of $30 \mathrm{mg} / \mathrm{m}^{2}$ compared with the $50 \mathrm{mg} / \mathrm{m}^{2}$ CAF regimen used in our validation sample. The rate of neutropenic events was markedly higher among the validation patients than in patients in the Silber study (84\% versus 47\%).

\section{Outcome definition}

The cross-validation of the Silber logistic regression model applied to our data produced a C-statistic of 0.78 . The 
Table 1

\begin{tabular}{|c|c|c|}
\hline Characteristic & $\begin{array}{c}\text { MDACC } \\
\text { study patients } \\
(1986-92) \\
(n=143)\end{array}$ & $\begin{array}{c}\text { Silber and } \\
\text { colleagues' patients } \\
(1985-93) \\
(n=95)\end{array}$ \\
\hline Mean age (years) & 46 (SD 10) & $46(\mathrm{SD} N / \mathrm{A})$ \\
\hline $\begin{array}{l}\text { Menopausal status }(\%) \\
\text { Pre- } \\
\text { Peri-, post, or surgical }\end{array}$ & $\begin{array}{l}55 \\
45\end{array}$ & $\begin{array}{l}60 \\
40\end{array}$ \\
\hline $\begin{array}{l}\text { Estrogen receptor status } \\
\text { Positive } \\
\text { Negative } \\
\text { Unknown }\end{array}$ & $\begin{array}{l}53 \\
37 \\
10\end{array}$ & $\begin{array}{c}47 \\
48 \\
4\end{array}$ \\
\hline $\begin{array}{l}\text { Progesterone receptor sta } \\
\text { Positive } \\
\text { Negative } \\
\text { Unknown }\end{array}$ & $\begin{array}{l}51 \\
36 \\
13\end{array}$ & $\begin{array}{l}48 \\
39 \\
13\end{array}$ \\
\hline $\begin{array}{l}\text { Tumor size }(\mathrm{cm})(\%) \\
\quad<2 \\
2-5 \\
>5 \\
\text { Unknown }\end{array}$ & $\begin{array}{l}36 \\
58 \\
6 \\
-\end{array}$ & $\begin{array}{c}41 \\
52 \\
4 \\
3\end{array}$ \\
\hline $\begin{array}{l}\text { Number of nodes (\%) } \\
\begin{array}{l}0 \\
1-3 \\
4-9 \\
>9 \\
\text { Unknown }\end{array}\end{array}$ & $\begin{array}{l}14 \\
61 \\
16 \\
9 \\
-\end{array}$ & $\begin{array}{l}- \\
76 \\
16 \\
5 \\
3\end{array}$ \\
\hline
\end{tabular}

All patient characteristics were insignificantly different between the two samples, except for the number of positive nodes. N/A, not available.

receiver-operating-characteristic curve for this analysis (Fig. 1) can be compared with the receiver-operating-characteristic curve from the original Silber model and an MDACC data-fitted model. The predictions obtained from the model for this independent set of patients confirm the validity of the Silber model. As occurs typically, the predictive power is not as high in a cross-validation as in the original model development $(C=0.83$ in the Silber model), but the cross-validation $C$-statistic is strong evidence for the usefulness of the model. The cross-validation optimal sensitivity and specificity rates (obtained for a cutoff probability of 0.45 ) were $66.6 \%$ and $73.3 \%$, respectively.

The model fitted to the MDACC data (Table 2) confirms the significance of the first-cycle nadir ANC effect $(P<0.0001)$. It was the only significant predictor of subsequent neutropenic events among those available in the validation sample, including age, menopausal status, receptor status, previous radiotherapy, and first-cycle hemoglobin decrease. The decrease in hemoglobin concentration from baseline to first-cycle nadir was only borderline significant $(P=0.0686)$. These results suggest that
Figure 1

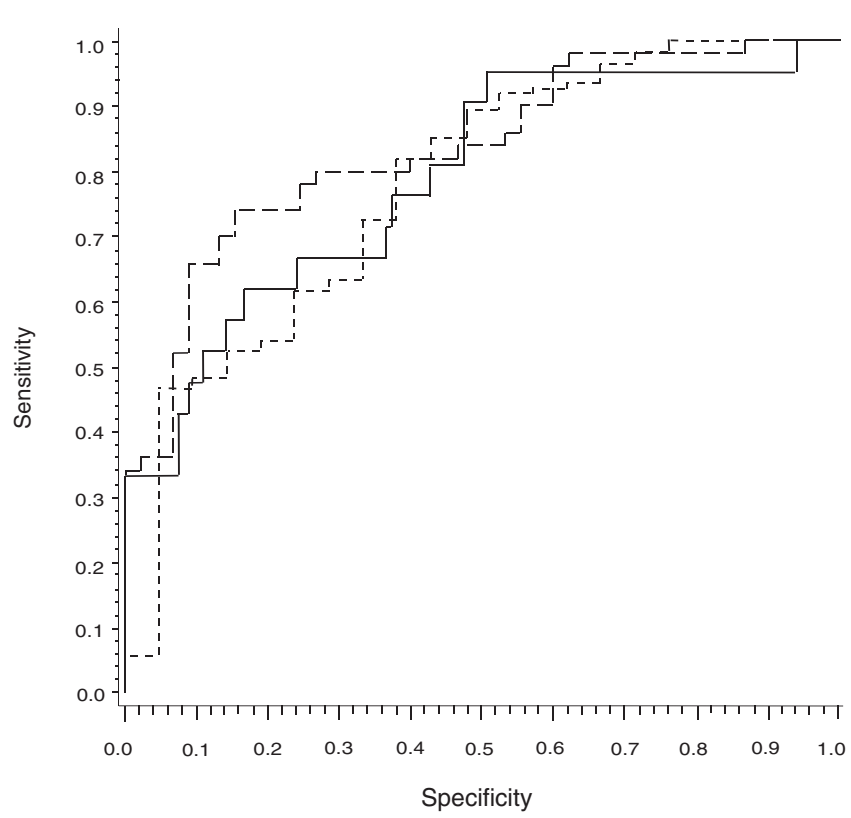

Comparison of receiver-operating-characteristic curves for multiple logistic regression models derived from the study by Silber and colleagues [1] $(n=95)$, the MDACC study $(n=143)$, and the cross-validation of the Silber model with the MDACC data. Dotted line, Silber model; broken line, MDACC data; solid line, cross-validation.

the first-cycle nadir ANC is an excellent predictor of neutropenic events in subsequent cycles. Model discrimination, as measured by the $\mathrm{C}$-statistic (or the area under the receiver-operating-characteristic curve), was similar in the original Silber and validation studies (0.83 versus 0.78 ). Figure 1 compares the receiver-operating-characteristic curves for the Silber and validation models. As with the Silber model, the validation model was well calibrated throughout the entire range of probabilities, as assessed by the nonsignificant Hosmer-Lemeshow statistic.

The MDACC model also confirms the magnitude of the effect. The estimated odds ratio of 4.8 for a unit $\left(1.0 \times 10^{9} /\right.$ liter $)$ decrease in first-cycle nadir ANC is not significantly different from 4.4 in the Silber study. An asymptotic $z$-test for the comparison of the estimated regression coefficients has a $P$ value of 0.91 . Estimates of first-cycle hemoglobin decrease effects were almost identical across models.

Model-derived point estimates for the probability of subsequent neutropenic events as a function of first-cycle nadir ANC and an average first-cycle decrease in hemoglobin concentration are presented in Fig. 2. The higher level of the estimated probability curve for the MDACC sample might be due to the higher myelotoxicity of the CAF regimen used in the validation sample. The observed pro- 
Table 2

Multiple logistic regression models for the validation and Silber and colleagues [1] studies

\begin{tabular}{|c|c|c|c|c|c|c|}
\hline \multirow[b]{2}{*}{ Variable } & \multicolumn{3}{|c|}{ MDACC study } & \multicolumn{3}{|c|}{ Silber model } \\
\hline & Odds ratio & $95 \% \mathrm{Cl}$ & $P$ & Odds ratio & $95 \% \mathrm{Cl}$ & $P$ \\
\hline Radiotherapy + chemotherapy indicator ${ }^{a}$ & - & & & 9.5 & $2.5-36.6$ & 0.0011 \\
\hline Cycle 1 nadir ANC ( $\times 10^{9} /$ liter $)$ & 4.8 & $2.3-9.9$ & $<0.0001$ & 4.4 & $2.1-9.2$ & $<0.0001$ \\
\hline Cycle 1 hemoglobin decrease (g/dl) & 1.8 & $1.0-3.3$ & 0.0686 & 1.8 & $1.2-2.8$ & 0.0074 \\
\hline$n$ & 143 & & & 95 & & \\
\hline Model $\chi^{2}$ & $21.2(2 \mathrm{df})$ & & & 30.3 (3 df) & & \\
\hline Log-likelihood $P$ & $<0.0001$ & & & $<0.0001$ & & \\
\hline C-statistic & 0.78 & & & 0.83 & & \\
\hline Hosmer-Lemeshow lack-of-fit $P$ & 0.1268 & & & 0.6140 & & \\
\hline
\end{tabular}

$\mathrm{Cl}$, confidence interval; df, degrees of freedom.

aNot applicable to MDACC study because patients were not treated with concurrent radiotherapy.

Figure 2

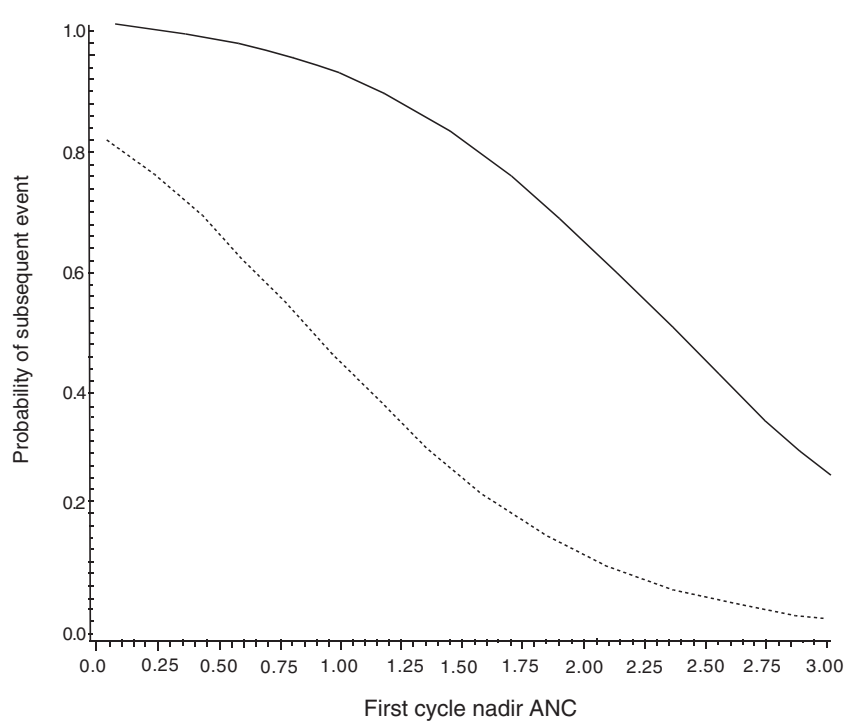

Probability of neutropenic-related event based on cycle 1 nadir ANC and average hemoglobin decrease at each sample. Point estimates derived from the Silber and MDACC multiple logistic regression models. Solid line, validation model; dotted line, Silber model.

portions of patients who experienced febrile neutropenia and received $85 \%$ or less of the planned dose intensity in the MDACC study are presented in Figs 3 and 4 by firstcycle nadir ANC. The association of the ANC at first-cycle nadir with each of the outcomes is evident. A significantly higher percentage of patients with a first-cycle nadir ANC of $0.25 \times 10^{9} /$ liter or less experienced febrile neutropenia (30\% versus $10 \%, P=0.04$ ) and received $85 \%$ or less of R118 the planned dose intensity ( $55 \%$ versus $32 \%, P=0.05)$.
Figure 3

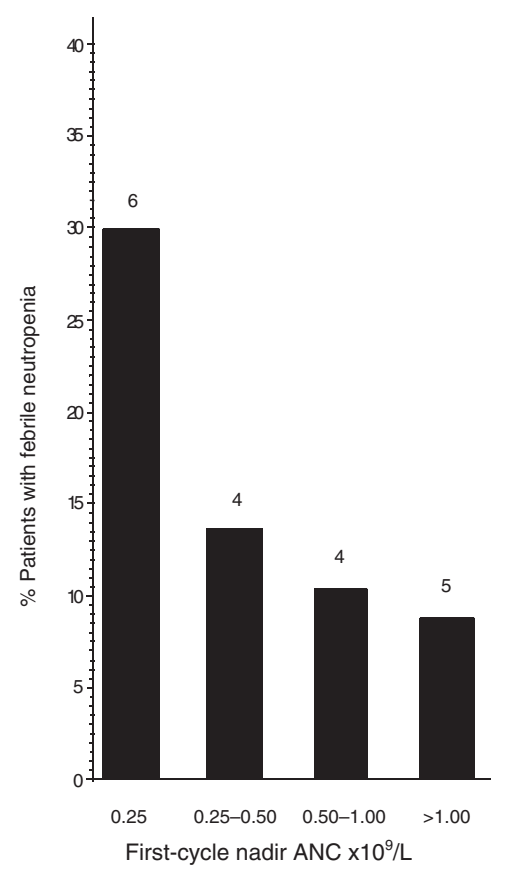

Rates of febrile neutropenia by first-cycle nadir ANC in the MDACC sample. Numbers of patients are shown above bars.

\section{Discussion}

Several retrospective analyses [1,2] using a variety of chemotherapies have consistently resulted in first-cycle nadir ANC as a primary predictor of neutropenia-related events in patients with breast cancer. Here we confirm the results obtained in the previous analyses by Silber and colleagues [1], which is important because the data used in this study come from a prospective clinical trial, 
Figure 4

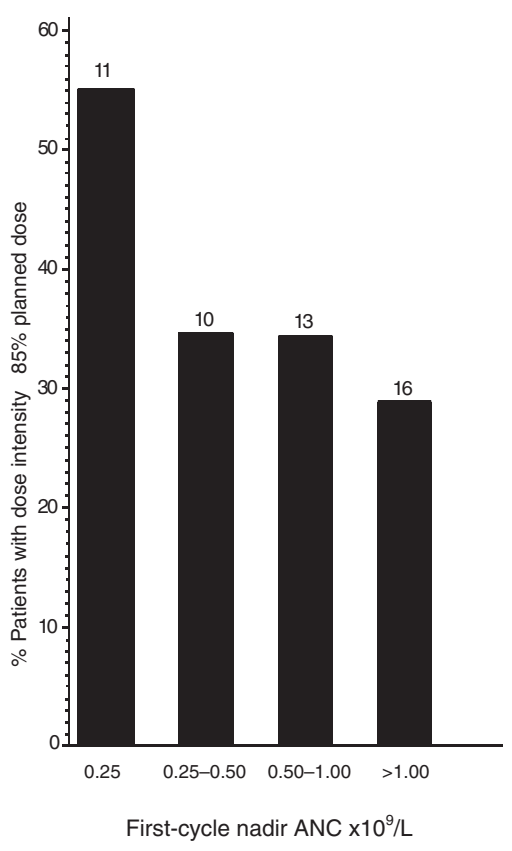

Rates of patients with a dose intensity not more than $85 \%$ of planned by first-cycle nadir ANC in the MDACC sample. Numbers of patients are shown above bars.

whereas the other data sets were collected from patient charts in an uncontrolled environment. The data in this study represent a homogenous population receiving standardized chemotherapy, and the collection procedures ensure high-quality data. The cross-validation analysis showed that the original predictive model successfully classified patients by their risk of neutropenic event. The model fitted to the validation data results in the same specification and similar variable coefficient magnitudes to those in the original model.

Our model differs somewhat from the Silber model. First, the impact of the change in hemoglobin concentration is significant in the Silber model and not significant in this data set, although the magnitude of the effect is similar in both models. We do not believe that hemoglobin concentrations have a direct impact on neutropenic complications; however, low hemoglobin concentrations probably provide further indication of bone marrow depletion. It is possible that the impact of change in hemoglobin concentration in the models will vary by chemotherapy regimen.

On the basis of the cumulated published evidence, the model seems to be robust. The model was tested in several chemotherapy regimens and in various clinical environments. It seems that the model's specification and the magnitude of the coefficient's effect is consistent across chemotherapy regimens. The inclination is to con- sider the implementation of this model prospectively. Before such a step is undertaken, some issues need to be discussed to improve our understanding of the model's limitation and its usefulness.

The major challenge to the model's prospective implementation is the uncertainty that a greater dose intensity would result in clinical benefit, such as survival. Although it is not within the scope of this study to answer this important question, it should be emphasized that the model provides some other distinctive and practical benefits. Successful application of the model will clearly define the patient group that should not receive growth-factor support and who can receive the full dose on time without a risk of complications. The ability to define the population that does not require growth factors on the basis of a quantitative clinical indicator has an immediate benefit to the patients and the health care system: it diverts resources from patients who will not benefit to those who will. The second benefit is the potential to reduce the morbidity due to febrile neutropenia from the high-risk group by providing these patients with growth-factor support before any clinical complications occur. The third benefit is learning the relationship between high dose intensity and survival on the basis of planned dose and not actual delivered dose. Implementation of a risk model can provide better data by making the planned dose closer to the delivered dose, and the impact of dose can be measured more accurately.

Additional objections to the model relate to the definition of a neutropenic complication, in particular dose delays and dose reductions. Dose delays and dose reductions are events that occur as a result of a clinical decision and not a measured biological variable such as neutropenia based on ANC. It could be argued that these decisions are not necessarily objective, reflecting the practice pattern of individual physicians, and cannot be part of the definition of a neutropenic complication. In this context it is important to remember that the purpose of the model is to identify patients at risk. A physician who delays or reduces the dose of chemotherapy has decided that the patient is at risk. Moreover, once it is decided to reduce or delay the dose, there is no way of knowing the rate of neutropenia or febrile neutropenia if the full dose of chemotherapy had been delivered. Therefore, including these events in the definition of a neutropenic complication seems justified, especially if the goal is to minimize harm to the patients. Admittedly, this methodology might result in an overestimation of the number of patients at risk.

Although the model seems to be robust and potentially useful, it does not answer the following two major questions. If patients were stratified by their risk for neutropenic complications and provided with growth-factor support, would the dose intensity delivered increase and by how much? Would the rate of neutropenic complications 
increase because of the higher chemotherapy dose delivered? To answer these questions decisively, the model needs to be implemented prospectively.

\section{Conclusions}

The first step toward the prospective implementation of the model has been performed and is reported by Rivera and colleagues [14]. Additional prospective studies on different regimens and different cancer types would be required to establish the utility and feasibility of this model. Such an effort carries with it the promise that clinical criteria would be used to deliver appropriate care to patients with cancer and would maximize the potential benefit from chemotherapy while possibly minimizing the burden on patients and their caregivers and families.

\section{Competing interests}

$\mathrm{MHE}$ is an employee of Amgen Inc., the manufacturer of filgrastim, and owns Amgen stocks and stock options. MF is a consultant to Amgen Inc. ER, DF, and GH have no competing interests.

\section{Acknowledgement}

MaryAnn Foote PhD assisted with the writing of the manuscript.

\section{References}

1. Silber JH, Fridman M, DiPaola RS, Erder MH, Pauly MV, Fox KR: First-cycle blood counts and subsequent neutropenia, dose reduction, or delay in early-stage breast cancer therapy. $J$ Clin Oncol 1998, 16:2392-2400.

2. Savvides P, Terrin, N, Erban J, Selker HR: Development and validation of a patient-specific predictive instrument for the need for dose-reduction in chemotherapy for breast cancer: a potential decision aid for the use of myeloid growth factors. Proc ASCO 2001, 20:244a.

3. Crawford J, Ozer H, Stoller R, Johnson D, Lyman G, Tabbara I, Kris M, Grous J, Picozzi V, Rausch G, Smith R, Gradishar W, Yahanda A, Vincent M, Stewart M, Glaspy J: Reduction by granulocyte colony-stimulating factor of fever and neutropenia induced by chemotherapy in patients with small-cell lung cancer. N Engl J Med 1991, 325:164-170.

4. de Graaf $\mathrm{H}$, Willemse PH, Bong SB, Piersma H, Tjabbes $\mathrm{T}$, van Veelen H, Coenen JL, deVries EG: Dose intensity of standard adjuvant CMF with granulocyte colony-stimulating factor premenopausal patients with node-positive breast cancer. Oncology 1996, 53:289-294.

5. Trillet-Lenoir V, Green J, Manegold C, Von Pawel J, Gatzemeier U, Lebeau B, Depierre A, Johnson P, Decoster G, Tomita D, Ewen C: Recombinant granulocyte colony-stimulating factor reduces the infection complications of cytotoxic chemotherapy. Eur $J$ Cancer 1993, 29A:319-324.

6. Link BK, Budd GT, Scott S, Dickman E, Paul D, Lawless G, Lee MW, Fridman M, Ford J, Carter WB, Oncology Practice Pattern Study Working Group: Delivering adjuvant chemotherapy to women with early-stage breast cancer: current patterns of care. Cancer 2001, 92:1354-1367.

7. Smith GA, Fine MJ, Lenert LL, Newbold RC: Project Chemolnsight reveals physician practice patterns for adjuvant breast cancer chemotherapy, and compares the dose intensity of CMF, AC, and CAF. Proc ASCO 1999, 18:78a.

8. Weiss, R, Valagussa, P, Moliterni A, Zambetti M, Buzzoni R, Bonadonna G: Adjuvant chemotherapy after conservative surgery plus irradiation versus modified radical mastectomy. Analysis of drug dosing and toxicity. Am J Med 1987, 83:455463.

9. Budman DR, Berry DA, Cirrincione CT, Henderson IC, Wood WC, Weiss RB, Ferree CR, Mus HB, Green MR, Norton L, Frei E: adjuvant treatment of breast cancer. The Cancer and Leukemia Group B. J Natl Cancer Inst 1998, 90:1205-1211.

10. Bonadonna G, Valagussa P, Moliterni A, Zambetti M, Brambilla C: Adjuvant cyclophosphamide, methotrexate, and fluorouracil in node-positive breast cancer: the results of $\mathbf{2 0}$ years of followup. N Engl J Med 1995, 332:901-906.

11. Silber JH, Fridman M, Shpilsky A, Even-Shoshan O, Smink DS, Jayaraman J, Fox KR, Pauly MV: Modeling the cost effectiveness of granulocyte colony-stimulating factor use in early-stage breast cancer. J Clin Oncol 1998, 16:2435-2444.

12. Rosner B: Fundamentals of Biostatistics, edn 5. Boston: PWSKent Publishing Company; 2000:63-65.

13. Hosmer DW, Lemeshow S: Applied Logistic Regression. New York: John Wiley \& Sons; 1989:271-274.

14. Rivera E: Targeted filgrastim support in patients with earlystage breast carcinoma. Cancer 2003, 98 in press.

\section{Correspondence}

Edgardo Rivera MD, University of Texas, MD Anderson Cancer Center, 1515 Holcombe Boulevard, Houston, TX 77030-0056, USA. Tel: +1 713 792 2817; fax: +1 713794 4385; e-mail: erivera@mail.mdanderson.org 